International Journal of Pure and Applied Mathematics

Volume 111 No. 1 2016, 129-139

ISSN: 1311-8080 (printed version); ISSN: 1314-3395 (on-line version)

url: http://www.ijpam.eu

doi: 10.12732 /ijpam.v111i1.12

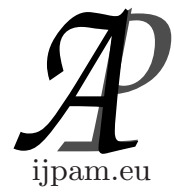

\title{
THE LEGENDRE POLYNOMIALS AS A BASIS FOR BESSEL FUNCTIONS
}

\author{
Clemente Cesarano ${ }^{1}$, Paolo Emilio Ricci ${ }^{2}$ \\ ${ }^{1,2}$ Section of Mathematics \\ Università Telematica Interazionale UNINETTUNO \\ Corso Vittorio Emanuele II, 39-Rome, ITALY
}

\begin{abstract}
By using the concepts and the formalism of the Monomiality Principle, we introduce a generalization of Bessel functions. The starting point is represented by Legendre polynomials, presented as particular case of generalized Laguerre polynomials of two variables. From the well known properties of the ordinary Bessel functions, we derive similar relations for this family of Legendre-Bessel functions, in particular, by exploiting the monomiality properties of Legendre polynomials, we discuss some differential rules of this family of generalized Bessel functions.
\end{abstract}

AMS Subject Classification: 33C10, 33C45, 30C99, 33B10

Key Words: Legendre polynomials, Bessel functions, Generating functions

\section{Introduction}

In a number of previous investigations ([1],[2], [3]) we have deeply discussed the concepts and the related formalism of the Monomiality Principle; in particular we have shown that it is possible to derive many operational identities for a wide class of orthogonal polynomials and special functions. The Monomiality Principle allows also us to state a different point of view regarding the properties of some families of polynomials, as for example Hermite ([4]) and Laguerre ([5]) polynomials and, moreover, to study isospectral problems as in the case of Hermite-Bessel functions and Laguerre-Bessel functions ([6]).

Received: $\quad$ October 23, 2016

Revised: $\quad$ November 29, 2016

Published: December 6, 2016

$\S$ Correspondence author (c) 2016 Academic Publications, Ltd.

url: www.acadpubl.eu 
We remind that the generalized Hermite polynomials $H_{n}(x, y)$ and twovariable Laguerre polynomials $L_{n}(x, y)$ can be viewed as quasi-monomials with the following operators $([2])$ :

$$
\begin{aligned}
\hat{M}_{H}=x+2 y \frac{\partial}{\partial x}, & \hat{M}_{L}=y-\hat{D}_{x}^{-1} \\
\hat{P}_{H}=\frac{\partial}{\partial x}, & \hat{P}_{L}=-\frac{\partial}{\partial x} x \frac{\partial}{\partial x} .
\end{aligned}
$$

Where we have denoted with $\hat{D}_{x}^{-1}$ the inverse of the derivative operator $([7])$. It has been shown that the Legendre polynomials ([8]) can be introduced directly by using the multiplication and derivative operators acting on Hermite and Laguerre polynomials, in the sense that we can combine the above operators (eq. (1)) to define new operators that can be used to view Legendre polynomials as quasi-monomials and at same time to define them. It is known that there exists a class of generalized Laguerre polynomials ([8],[9]) with the following explicit form:

$$
{ }_{2} L_{n}(x, y)=n ! \sum_{r=0}^{\left[\frac{\mathrm{n}}{2}\right]} \frac{x^{r} y^{n-2 r}}{(n-2 r) !(r !)^{2}}
$$

where:

$$
{ }_{2} L_{0}(x, y)=1
$$

By introducing the operators:

$$
\begin{aligned}
\hat{M} & =y+2 \hat{D}_{x}^{-1} \frac{\partial}{\partial y}, \\
\hat{P} & =\frac{\partial}{\partial y},
\end{aligned}
$$

after noting that:

$$
[\hat{M}, \hat{P}]=1,
$$

we can immediately write that:

$$
\hat{M}^{n}(1)=\left(y+2 \hat{D}_{x}^{-1} \frac{\partial}{\partial y}\right)^{n}(1)=n ! \sum_{r=0}^{\left[\frac{\mathrm{n}}{2}\right]} \frac{x^{r} y^{n-2 r}}{(n-2 r) !(r !)^{2}}
$$

where we have used the identity:

$$
\hat{D}_{x}^{-n}=\frac{x^{n}}{n !} .
$$


These generalized Laguerre polynomials can be reduced to the ordinary Legendre polynomials:

$$
P_{n}(x)=n ! \sum_{r=0}^{\left[\frac{\mathrm{n}}{2}\right]} \frac{(-1)^{n-2 r} x^{r}\left(1-x^{2}\right)^{n-2 r}}{(n-2 r) !(r !)^{2} 2^{2(n-2 r)}}
$$

by setting:

$$
{ }_{2} L_{n}\left(-\frac{1}{4}\left(1-x^{2}\right), x\right)=P_{n}(x) .
$$

By using the formalism of Monomiality Principle ([1]), we can also derive the generating function of the above polynomials. Since we have observed that ${ }_{2} L_{0}(x, y)=1$, we immediately get:

$$
\exp (t \hat{M})(1)=\sum_{n=0}^{+\infty} \frac{t^{n}}{n !}{ }_{2} L_{n}(x, y)
$$

where $t$ is a continuous parameter. By exploiting the exponential on the above relation, we have:

$$
\exp (t \hat{M})(1)=\exp \left[t\left(y+2 \hat{D}_{x}^{-1} \frac{\partial}{\partial y}\right)\right](1)=\exp \left(t y+2 t \hat{D}_{x}^{-1} \frac{\partial}{\partial y}\right)(1)
$$

Since the operators $t y$ and $2 t \hat{D}_{x}^{-1} \frac{\partial}{\partial y}$ do not commute, we have to compute their commutator:

$$
\left[t y, 2 t \hat{D}_{x}^{-1} \frac{\partial}{\partial y}\right]=-2 t^{2} \hat{D}_{x}^{-1}
$$

and then equation (10) could be written as:

$$
\exp \left[t\left(y+2 \hat{D}_{x}^{-1} \frac{\partial}{\partial y}\right)\right](1)=\exp (t y) \exp \left(2 \hat{D}_{x}^{-1} \frac{\partial}{\partial y}\right) \exp \left(t^{2} \hat{D}_{x}^{-1}\right)(1)
$$

or, in a more convenient form:

$$
\exp \left[t\left(y+2 \hat{D}_{x}^{-1} \frac{\partial}{\partial y}\right)\right](1)=\exp (t y) \exp \left(t^{2} \hat{D}_{x}^{-1}\right) \exp \left(2 \hat{D}_{x}^{-1} \frac{\partial}{\partial y}\right)(1)
$$

From the fact that:

$$
\exp \left(2 \hat{D}_{x}^{-1} \frac{\partial}{\partial y}\right)(1)=1
$$


we finally obtain:

$$
e^{y t} e^{\hat{D}_{\times}^{-1} t^{2}}=\sum_{n=0}^{+\infty} \frac{t^{n}}{n !}{ }_{2} L_{n}(x, y),
$$

that is the generating function of generalized Laguerre polynomials ${ }_{2} L_{n}(x, y)$. It is immediately noted that $([7])$ :

$$
{ }_{2} L_{n}(x, y)=H_{n}\left(y, \hat{D}_{x}^{-1}\right)
$$

to outline the common nature of the monomiality operators used, relatively to Hermite and Laguerre polynomials families. The Monomiality Principle operational techniques exploited for other classes of polynomials, as reminder for Hermite and Laguerre families, suggest to state further relations for the polynomials ${ }_{2} L_{n}(x, y)$. Directly from equations (3), we have:

$$
\begin{aligned}
& \hat{M}\left(=y+2 \hat{D}_{x}^{-1} \frac{\partial}{\partial y}\right){ }_{2} L_{n}(x, y)={ }_{2} L_{n+1}(x, y), \\
& \hat{P}\left(=\frac{\partial}{\partial y}\right){ }_{2} L_{n}(x, y)=n_{2} L_{n-1}(x, y),
\end{aligned}
$$

to allow us to state the following differential equation solved by polynomials ${ }_{2} L_{n}(x, y)$ :

$$
\hat{M} \hat{P}_{2} L_{n}(x, y)=n_{2} L_{n}(x, y),
$$

or in differential form:

$$
\left(y+2 \hat{D}_{x}^{-1} \frac{\partial}{\partial y}\right)\left(\frac{\partial}{\partial y}\right){ }_{2} L_{n}(x, y)=n_{2} L_{n}(x, y)
$$

that gives:

$$
\left(y+2 \hat{D}_{x}^{-1} \frac{\partial}{\partial y}\right)\left(\frac{\partial}{\partial y}\right){ }_{2} L_{n}(x, y)=n_{2} L_{n}(x, y) .
$$

Furthermore, we can also observe that the Tricomi function of order zero, of a suitable variable, reads:

$$
C_{0}\left(-x t^{2}\right)=\sum_{n=0}^{+\infty} \frac{t^{2 n}}{n !} \frac{x^{n}}{n !}
$$

and then, from equation (15), we can write a different form of the generating function of polynomials ${ }_{2} L_{n}(x, y)$ :

$$
e^{y t} C_{0}\left(-x t^{2}\right)=\sum_{n=0}^{+\infty} \frac{t^{n}}{n !}{ }_{2} L_{n}(x, y)
$$


and, in terms of ordinary Legendre polynomials:

$$
\sum_{n=0}^{+\infty} \frac{t^{n}}{n !} P_{n}(x)=e^{x t} C_{0}\left(\frac{1}{4}\left(1-x^{2}\right) t^{2}\right) .
$$

The Legendre polynomials presented in this section in terms of the special class of the generalized Laguerre polynomials of type ${ }_{2} L_{n}(x, y)$, could be used to introduce a further generalization of the p-based Bessel functions as discussed in the previous papers $([6],[10])$. In the following sections we will discuss the aspects and the related properties of the Legendre-Bessel functions.

\section{Legendre-Bessel Functions}

It has been shown that, in the framework of the monomiality principle, it is possible to construct a class of Bessel-type functions, by using different families of polynomials, recognized as quasi-monomial. In particular, we have discussed the properties of the Laguerre-Bessel functions ([10]):

$$
{ }_{p} J_{n}(x)=\sum_{n=0}^{+\infty} \frac{(-1)^{r} x^{n+2 r}}{2^{n+2 r} r !(n+r) !(n+2 r) !}
$$

and we have obtained some useful operational results, as for example the following differential equation, solved by the Bessel functions of type ${ }_{p} J_{n}(x)$ :

$$
\left[\left(\frac{d}{d x} x \frac{d}{d x}\right)^{2}+\frac{d}{d x}\left(\frac{d}{d x} x \frac{d}{d x}\right)-n^{2} \frac{d^{2}}{d x^{2}}+1\right]{ }_{p} J_{n}(x)=0 .
$$

In the previous section, we have introduced a generalized family of Laguerre polynomials that could be treated as ordinary Legendre polynomials see eq. (...); we use these polynomials to introduce a further class of Bessel-type functions, by following an operational approach in according with the definition and the related techniques of monomiality principle.

We start to consider the classical first kind Bessel function ([5]), defined as follows:

$$
J_{n}(x)=\sum_{n=0}^{+\infty} \frac{(-1)^{r} x^{n+2 r}}{2^{n+2 r} r !(n+r) !},
$$

where $r$ is an integer, and the generating function is given by:

$$
\exp \left[\frac{x}{2}\left(t-\frac{1}{t}\right)\right]=\sum_{n=0}^{+\infty} t^{n} J_{n}(x) .
$$


By applying the differential isomorphism to the two-variable Laguerre polynomials of type ${ }_{2} L_{n}(x, y)$, we have:

$$
\begin{gathered}
\hat{M} \rightarrow x, \\
\hat{P} \rightarrow \frac{\partial}{\partial y}, \\
{ }_{2} L_{n}(x, y) \rightarrow x^{n},
\end{gathered}
$$

where $\hat{M}=y+2 \hat{D}_{x}^{-1} \frac{\partial}{\partial y}$ and $\hat{P}=\frac{\partial}{\partial y}$.

From relation $(28)_{1}$, by substituting the isospectral values, we formally get:

$$
\exp \left[\frac{\hat{M}}{2}\left(t-\frac{1}{t}\right)\right]=\sum_{n=0}^{+\infty} t^{n}{ }_{L} J_{n}(x, y)
$$

where ${ }_{L} J_{n}(x, y)$ denotes the Legendre-Bessel function to be defined.

By expliciting the exponential on the above equation, we have:

$$
\exp \left[\frac{\hat{M}}{2}\left(t-\frac{1}{t}\right)\right]=\exp \left[\frac{1}{2}\left(y+2 \hat{D}_{x}^{-1} \frac{\partial}{\partial y}\right)\left(t-\frac{1}{t}\right)\right]
$$

that is:

$$
\exp \left[\frac{\hat{M}}{2}\left(t-\frac{1}{t}\right)\right]=\exp \left[\frac{y}{2}\left(t-\frac{1}{t}\right)+\hat{D}_{x}^{-1} \frac{\partial}{\partial y}\left(t-\frac{1}{t}\right)\right] .
$$

Since the two operators

$$
\hat{A}:=\frac{y}{2}\left(t-\frac{1}{t}\right)
$$

and

$$
\hat{B}:=\hat{D}_{x}^{-1} \frac{\partial}{\partial y}\left(t-\frac{1}{t}\right)
$$

do not commute, it is necessary to compute their commutator ([11]):

$$
[\hat{A}, \hat{B}]=-2 \hat{D}_{x}^{-1}\left(t-\frac{1}{t}\right)^{2}
$$


We can now rewrite the relation (31) in the following way:

$$
\begin{aligned}
& \exp \left[\frac{\hat{M}}{2}\left(t-\frac{1}{t}\right)\right]= \\
& =\exp \left[\frac{y}{2}\left(t-\frac{1}{t}\right)\right] \exp \left[\hat{D}_{x}^{-1} \frac{\partial}{\partial y}\left(t-\frac{1}{t}\right)\right] \exp \left[\frac{1}{4} \hat{D}_{x}^{-1}\left(t-\frac{1}{t}\right)^{2}\right]
\end{aligned}
$$

and by exploiting the fact that the exponentials now commute, we have:

$$
\exp \left[\frac{\hat{M}}{2}\left(t-\frac{1}{t}\right)\right]=\exp \left[\frac{y}{2}\left(t-\frac{1}{t}\right)+\frac{1}{4} \hat{D}_{x}^{-1}\left(t-\frac{1}{t}\right)^{2}\right],
$$

after noting that:

$$
\exp \left[2 \hat{D}_{x}^{-1} \frac{\partial}{\partial y}\left(t-\frac{1}{t}\right)\right](1)=1
$$

The relation in equation (29) represents the generating function of the Legendre-Bessel function ${ }_{L} J_{n}(x, y)$.

It could be useful to note that, by setting:

$$
\frac{1}{2}\left(t-\frac{1}{t}\right)=\bar{t}
$$

the relation in equation (29) becomes:

$$
\exp \left[\frac{\hat{M}}{2}\left(t-\frac{1}{t}\right)\right]=\exp \left[y \bar{t}+\hat{D}_{x}^{-1} \bar{t}^{2}\right]=\sum_{n=0}^{+\infty} \frac{\bar{t}^{n}}{n !} H_{n}\left(y, \hat{D}_{x}^{-1}\right)
$$

and, after noting that Laguerre polynomials of type ${ }_{2} L_{n}(x, y)$ are linked to generalized two-variable Hermite polynomials ([7]) by the following relation:

$$
{ }_{2} L_{n}(x, y)=H_{n}\left(y, \hat{D}_{x}^{-1}\right)
$$

we can also write

$$
\exp \left[\frac{\hat{M}}{2}\left(t-\frac{1}{t}\right)\right]=\sum_{n=0}^{+\infty} \frac{\bar{t}^{n}}{n !}{ }_{2} L_{n}(x, y)
$$

that outlines the nature of Legendre-Bessel functions. 
By expliciting the r.h.s. of equation (39), we have:

$$
\begin{aligned}
\sum_{n=0}^{+\infty} \frac{\bar{t}^{n}}{n !} H_{n}\left(y, \hat{D}_{x}^{-1}\right) & =\sum_{n=0}^{+\infty} \frac{\left(t-\frac{1}{t}\right)^{n}}{2^{n} n !} H_{n}\left(y, \hat{D}_{x}^{-1}\right) \\
& =\sum_{n=0}^{+\infty} \sum_{s=0}^{n}\left(\begin{array}{l}
n \\
s
\end{array}\right) \frac{(-1)^{s}}{2^{n} n !} t^{n-2 s} H_{n}\left(y, \hat{D}_{x}^{-1}\right)
\end{aligned}
$$

and, after setting $m=n-2 s$, we get:

$$
\sum_{n=0}^{+\infty} \frac{\bar{t}^{n}}{n !} H_{n}\left(y, \hat{D}_{x}^{-1}\right)=\sum_{m=0}^{+\infty} t^{m} \sum_{s=0}^{+\infty} \frac{(-1)^{s}}{2^{m+2 s}(m+s) ! s !} H_{m+2 s}\left(y, \hat{D}_{x}^{-1}\right)
$$

that allows us to state the explicit form of generalized Bessel function on Legendre basis:

$$
{ }_{L} J_{n}(x, y)=\sum_{s=0}^{+\infty} \frac{(-1)^{s}}{2^{n+2 s}(n+s) ! s !} H_{n+2 s}\left(y, \hat{D}_{x}^{-1}\right)
$$

or, that is the same (eq. (...)):

$$
{ }_{L} J_{n}(x, y)=\sum_{s=0}^{+\infty} \frac{(-1)^{s}}{2^{n+2 s}(n+s) ! s !}{ }_{2} L_{n+2 s}(x, y) \text {. }
$$

We can also deduce the explicit form of the above Legendre-Bessel function, by expanding directly their generating function, derived above (see eq. (39)). We have, in fact:

$$
\begin{aligned}
\exp \left[\frac{y}{2}\left(t-\frac{1}{t}\right)+\frac{1}{4} \hat{D}_{x}^{-1}\left(t-\frac{1}{t}\right)^{2}\right]= \\
=\exp \left[\frac{y}{2}\left(t-\frac{1}{t}\right)\right] \exp \left[\frac{1}{4} \hat{D}_{x}^{-1}\left(t-\frac{1}{t}\right)\right] \\
=\sum_{m=0}^{+\infty} t^{m} J_{m}(y) \sum_{s=0}^{+\infty} t^{2 s} J_{s}\left(\frac{\hat{D}_{x}^{-1}}{2}\right)
\end{aligned}
$$

and then:

$$
{ }_{L} J_{n}(x, y)=\sum_{s=-\infty}^{+\infty} J_{n-2 s}(y) J_{s}\left(\frac{\hat{D}_{x}^{-1}}{2}\right)
$$


Furthermore, by noting that:

$$
J_{s}\left(\frac{\hat{D}_{x}^{-1}}{2}\right)=\sum_{s=-\infty}^{+\infty} \frac{(-1)^{s}}{(s+r) ! r !}\left(\frac{\hat{D}_{x}^{-1}}{2}\right)^{s+2 r}
$$

and by considering that

$$
\hat{D}_{x}^{-(s+2 r)}=\frac{x^{s+2 r}}{2^{s+2 r}(s+2 r) !}
$$

we can conclude with:

$$
J_{s}\left(\frac{\hat{D}_{x}^{-1}}{2}\right)=\sum_{s=-\infty}^{+\infty} \frac{(-1)^{s}}{2^{s+2 r}(s+r) ! r !(s+2 r) !} x^{s+2 r} .
$$

\section{Operational Results}

We have introduced in the previous section the generalized Legendre-Bessel functions and we have stated their generating function (see eq. (29)). We can now observe how is possible to derive some useful results, by using the similar relations satisfied by the two-variable, generalized Bessel functions. The focus is essentially to draw the approach to use when we study isospectral problems by using the formalism of Monomiality Principle; we are indeed interested to outline the techniques contained in the method of Monomiality Principle. From the generating function of Legendre-Bessel functions ${ }_{L} J_{n}(x, y)$

$$
\begin{aligned}
\exp \left[\frac{\hat{M}}{2}\left(t-\frac{1}{t}\right)\right] & =\exp \left[\frac{y}{2}\left(t-\frac{1}{t}\right)+\frac{1}{4} \hat{D}_{x}^{-1}\left(t-\frac{1}{t}\right)^{2}\right] \\
& =\sum_{n=0}^{+\infty} t^{n}{ }_{L} J_{n}(x, y)
\end{aligned}
$$

by deriving, with respect to $t$, both sides of above equation:

$$
\frac{\hat{M}}{2}\left(1+\frac{1}{t^{2}}\right) \exp \left[\frac{\hat{M}}{2}\left(t-\frac{1}{t}\right)\right]=\sum_{n=0}^{+\infty} n t^{n-1}{ }_{L} J_{n}(x, y)
$$

and by expanding the 1.h.s in equation (52), we get:

$$
\frac{\hat{M}}{2}\left[\sum_{n=0}^{+\infty} t^{n}{ }_{L} J_{n}(x, y)+\sum_{n=0}^{+\infty} t^{n-2}{ }_{L} J_{n}(x, y)\right]=\sum_{n=0}^{+\infty} n t^{n-1}{ }_{L} J_{n}(x, y)
$$


that is, after rearranging the indexes:

$$
\frac{\hat{M}}{2}\left[\sum_{n=0}^{+\infty} t^{n+1}{ }_{L} J_{n}(x, y)+\sum_{n=0}^{+\infty} t^{n-1}{ }_{L} J_{n}(x, y)\right]=\sum_{n=0}^{+\infty} n t^{n}{ }_{L} J_{n}(x, y),
$$

which allows us to state the following recurrence relation for the generalized Legendre-Bessel functions:

$$
\frac{\hat{M}}{2}\left[{ }_{L} J_{n-1}(x, y)+{ }_{L} J_{n+1}(x, y)\right]=n_{L} J_{n}(x, y) .
$$

The recurrence relations stated above can be used to derive further interesting operational results for this kind of generalized Bessel functions, based on Legendre polynomials, that we will discuss in a forthcoming paper. It could also be noted that Legendre-Bessel functions when $x=0$ reduce to (see equation $(45))$ :

$$
{ }_{L} J_{n}(0, y)=\sum_{s=0}^{+\infty} \frac{(-1)^{s}}{2^{n+2 s}(n+s) ! s !}{ }_{2} L_{n+2 s}(0, y)
$$

and by noting the value of the polynomials of type ${ }_{2} L_{n}(x, y)$ when $x=0$, allow us to explore different operational relations involving this kind of Bessel functions. The concepts and the related formalism exploited in this paper to construct interested generalizations of Bessel functions has been used to derive similar results by using as basis the Hermite polynomials (see [12], [10]), but in general it is possible to extend the procedure focusing on other families of polynomials, recognized as quasi-monomials as for instance generalized classes of Hermite polynomials (see [3], [13], [14]) or families belonging to the class of Chebyshev polynomials (see [15], [16], [17], [18]).

\section{References}

[1] C. Cesarano, G.M. Cennamo, L. Placidi, Operational methods for Hermite polynomials with applications, WSEAS Transaction on Mathematics, 13 (2014), 925-931.

[2] Y. Ben Cheik, Some results on quasi-monomiality, Applied Mathematics and Computation, 141(1) (2003), 63-76.

[3] C. Cesarano, C. Fornaro, L. Vazquez, Operational results on bi-orthogonal Hermite functions, Acta Mathematica Universitatis Comenianae, 85(1) (2016), 43-68.

[4] P. Appell, J. Kampé de Fériet, Fonctions hypergéométriques et hypersphériques. Polinomes d'Hermite, Gauthier-Villars, Paris (1926). 
[5] M. Abramowitz, I.A. Stegun, Handbook of Mathematical Functions with Formulas, Graphs, and Mathematical Tables, Dover Publications, Inc., New York (1972).

[6] G. Dattoli, H.M. Srivastava, D. Sacchetti, The Hermite polynomials and the Bessel functions from a general point of view, International Journal of Mathematics and Mathematical Sciences, 57 (2003), 3633-3642.

[7] G. Dattoli, S. Lorenzutta, P.E. Ricci, C. Cesarano, On a family of hybrid polynomials, Integral Transforms and Special Functions, 15(6) (2004), 485-490.

[8] G. Dattoli, H.M. Srivastava, C. Cesarano, The Laguerre and Legendre polynomials from an operational point of view, Applied Mathematics and Computation, 124(1) (2001), $117-127$.

[9] G. Dattoli, P.E. Ricci, C. Cesarano, A note on Legendre polynomials, International Journal of Nonlinear Sciences and Numerical Simulation, 2(4) (2001), 365-370.

[10] C. Cesarano, B. Germano, P.E. Ricci, Laguerre-type Bessel functions, Integral Transforms and Special Functions, 16(4) (2005), 315-322.

[11] W. Miller, Lie Theory and Special functions, Academic Press, New York, (1968).

[12] G. Dattoli, Comments on the theory of Hermite-Bessel functions, Integral Transforms and Special Functions, 15(4) (2004), 303-308.

[13] D. Assante, C. Cesarano, C. Fornaro, L. Vazquez, Higher order and fractional diffusive equations, Journal of Engineering Science and Technology Review, 8(5) (2015), 202-204.

[14] C. Cesarano, C. Fornaro, L. Vazquez, A note on a special class of Hermite polynomials, International Journal of Pure and Applied Mathematics, 98(2) (2015), 261-273.

[15] C. Cesarano, Integral representations and new generating functions of Chebyshev polynomials, Hacettepe Journal of Mathematics and Statistics, 44(3) (2015), 535-546.

[16] C. Cesarano, Generalized Chebyshev polynomials, Hacettepe Journal of Mathematics and Statistics, 43(5) (2014), 731-740.

[17] C. Cesarano, C. Fornaro, Operational identities on generalirized two-variable Chebyshev polynomials, International Journal of Pure and Applied Mathematics, 100(1) (2015), 59-74.

[18] C. Cesarano, Identities and generating functions on Chebyshev polynomials, Georgian Mathematical Journal, 19(3) (2012), 427-440. 
